\title{
Research on the Forming Module of Bagging Machine Fei ZHONG ${ }^{a}$, Wen-Ding $\mathrm{LI}^{\mathrm{b}}$, Zhen HUANG ${ }^{\mathrm{c}}$, and Xin WANG ${ }^{\mathrm{d}}$ Hubei University of Technology, No.28, Nanli Road, Hong-shan District, Wuchang, Wuhan, Hubei Province, P. R. China 430068
}

\author{
491693789@qq.com
}

Keywords: Solid works modeling, Mechanical design, Pneumatic system, Servo system, Intelligent.

\begin{abstract}
In this paper, after understanding the relevant circumstances the of the cooperative enterprise packaging bag production, the bagging mechanism of the mechanical forming has been studied. The structure is modeled with SolidWorks, and the mechanical principle and mechanical design are used to realize the automatic production of the inner bag and improve the production efficiency. Pneumatic system selection cylinder, piston rod, after the data obtained with the body of the most suitable match. Servo system Based on the pneumatic system, the servo system inertia, diameter, speed, torque calculation is selected, select the optimization parameters. Based on the above design calculations, to improve the efficiency of packaging institutions to make a worthwhile exploration. Which based on the theory of aerodynamic research and application of pneumatic theory in the mechanical design has contributed to the application.
\end{abstract}

\section{Introduction}

In this paper, it can be realized that automatic, efficient, stable and flexible automatic bagging machine, which can work in complicated environment and realize the automation of supplying bags, grabbing bags, extending bags, sack, sewing, collecting bags. Based on the requirement of bagging automatic machine, design the object and task, use solid works to design all parts of bagging machine, and then study the mechanism.

\section{Goals of Packing Automatic Molding Machine Design}

According to the design requirements of bagging automatic molding machine, the machine imitates artificial bagging machine movements and it needs to supply bags, grabbing bags, extending bags, sack, sewing, collecting bags an a series of actions.

According to the above-mentioned design requirements, based on the mechanical design concept of improving the efficiency, reliability and reducing the cost, the design principle of the sleeve film machine is put forward.

(1)As far as possible to meet the requirements of manufacturers, the use of simple institutions and components, size distribution is reasonable, simple motion control;

(2)The location of bagging is accurate, and sets of film machine mechanical structure is compact

(3)The establishment of bagging machine three-dimensional model, check the parts interference;.

\section{Requirements of the Whole Structure}

Set of film packaging machine bag automatic production line raw material is thin nylon bag and leather bag outside the paper, that is, by the inner pocket of the bag into the bag accurately, and then complete the sewing. Automatic sleeve film machine imitation artificial bagging action to automatically take the bag, to send bags, bagging, sewing, into a series of bags and other actions. According to the function of the mechanism, the mechanical system of the inner sleeve filming machine is divided into a bag feeding system, a bagging system, a bagging system, a sewing system, a bottom sewing system and a bag collecting system.

By examining the plant-specific production environment, the system functions are as follows: 
(1)When the bag is piled up, the device can have an automatic bag-catching device, taking into account the need for intermittent outer bags, designed for the switch of the two silos, in front of the use of a conveyor device to provide bag.

(2)Put the bag into the bag before puting outside, inner and ouer bag and the robot must have a precise positional relationship, so the outer bag must take a fixed length, the accuracy of the requirements must meet the standards.

(3)Sewing the previous process is bagging, the later process is into the bag, the speed of bags being sent to the sewing and the sewing machine should be able to match。

\section{Mechanism Design}

There are the following system components in the automatic bagging machin: a bag feeding mechanism, a bag feeding mechanism, a feeding mechanism, a bagging mechanism, a sewing mechanism, a sewing mechanism and a bag collecting mechanism. When the system is working, require close coordination of agencies, convergence of unity.

The main function of the storage bag system is to store empty bags, bags can be carried by workers on the bag, automatic bag system to take bags to take place. For the bag system design, the following requirements need to be meer:

Bag storage system's main function is to store empty bags, bags can be moved to the station by workers, and automatic to the location of taking bag system .The design of supplying bag system needs to meet the following requirements:

(1)The bag-feeding station can complete the bag-storing function, and can store a plurality of manually placed bags;

(2)The bag can be moved from the supplying bag station to the taking bag station;

(3)The mechanism taking the bag from the workplace to is be equipped with step-by-step movement, when the bags were removed in turn, the body can move to the location of the target bag to make up position;

(4)The bag storage system needs to have features that the empty bag be examed, and takes the bag goal position examination.

The working principle is: the feeding mechanism has two storage bins outside the bag, used to switch the feeding station. When the outer bag in a silo is finished, the lateral cylinder below the silo will move to realize the switching of the silo, so as to realize the uninterrupted process of the feeding process, so as to finish the bag process successfully.

The bag-taking system takes the bag from the bag-feeding system and delivers the bag to the bagging station. Take bag to send bag is bagging of the preparation steps. Take the bag system design needs to meet the following requirements:

(1) Ensure that the it is herizontal before it is bagged;

(2) The bag system will be sent to the bag in accurate position.

With the three-phase asynchronous motor providing power for the timing belt timing belt, and pressure roller together to complete the transfer of the outer bag. The vacuum disk and the 180 degree air paw can be used to suck and hold the outer bag. At the same time, the cylinder-driven pressure wheel can speed up the bag outside the transport speed, transmission speed more stable.

Bagging mechanism is composed of servo manipulator, open bag cylinder, vacuum negative pressure system and rotary cylinder. On the bag, the whole bag to complete the function of the station to be: the bag from the silo to move to the transfer mechanism, the transfer body to complete the finishing position of the outer bag for the bag to reach the bag site to prepare accurate. In the feeding section, with the cylinder to control the vacuum chuck up and down before and after the action to complete the outer bag from the silo to move the purpose of the conveyor belt. And then push the side of the bag by controlling the side of the baffle to control the location of the outer bag, the outer bag being ready for finishing the next step to open bag bagging.

The main function of the sending sewing mechanism is to take the packing bag of the inner bag from the stopper mechanism and transport it to the sewing machine. The design needs to meet the 
following requirements:

(1) The process of moving bags to ensure that the bag sets a good bag and ensure the attitude of the transport process level;

(2) The process of moving bags to move quickly and accurately;

(3) Transfer bag to ensure that the pocket formation.

When the bags are opened, the bags are clamped on both sides of the bags, which can ensure the level of the bags during transportation.

Sewing mechanism is the key step after bagging is completed by the sewing machine will be permanently fixed inside and outside the bag together to complete the production of composite bags. One of the sewing machine control is the key to the quality of finished products.

The working principle is: after the bag finished the compound bag, will be sent to the sewing machine into the sewing machine through the sewing machine. The sewing machine is started. Completion of a sewing station after completion.

The inner bag feeding mechanism is composed of a bag making machine, a servo motor, a rotary cylinder and a frame.

The working principle is that the inner bag will be made when the two bags in the inner bag platform do not have the inner bag, and the first inner bag made will be transported to another station through the folder bag cylinder, When the bags are in the station, they will wait for the robot to issue a grab action.

The bag collecting mechanism is mainly composed of a conveying device, a cylinder and the like, which are composed of a frame, a bag retaining part, a transmission shaft and a transmission belt.

Working principle: When the motor is driven by the transmission, the drive shaft rotates counterclockwise, and the sprocket provided at both ends is rotated to drive the chain rotation. The mechanical claw fixed on the chain rotates counterclockwise, and when the rotation lever passes through the cam provided on the fixed shaft, the jaws are opened quickly to catch the sealed bags. When the pulley on the top of the handle is disengaged from the cam, the jaws automatically clamp the bag counter-clockwise to the bag-releasing device. The rotating lever is separated from the front of the bag-releasing device. Cam quickly put down the bag in the specified position, the entire action is complete. So complete bag function back and forth. After receiving 50 bags, the cylinder set in the lower part of the rack will put the bag cover into the bottom of the belt level, the control motor to start the transmission belt will set the woven bag conveyor to the end of the rack to facilitate the workers Bale.

\section{Pneumatic Part}

Set of automatic film forming machine gas includes: air source part of the gas, the cylinder circuit design, and vacuum suction circuit part. In the head and tail of the cylinder it needs to add a magnetic switch, used to detect the output signal, control sets of film machine running. Vacuum sucker part of the air sucker selection and vacuum mode, air source part of the design are based on the maximum air consumption and the average air consumption to select the air compressor. Maximum air volume calculation formula:

$$
q_{r}=0.0462 D^{2} u_{m}(p+0.102)
$$

Where: $\mathrm{D}$ is the bore, in $\mathrm{cm}$; $\mathrm{u}_{\mathrm{m}}$ for the maximum piston speed, the unit is $\mathrm{mm} / \mathrm{s} ; \mathrm{p}$ is the pressure, the unit is MP.

Calculation formula of average gas:

$$
q_{r}=0.0157 N D^{2} s(p+0.1) / 0.1
$$

Where: $\mathrm{N}$ is the number of reciprocating piston rod; $\mathrm{s}$ for the cylinder, in units of $\mathrm{cm}$.

Vacuum chuck design: Vacuum chuck has been widely used in the field of automatic packaging. Along with the innovation and development of pneumatic technology, the performance of vacuum sucker is also changing with each passing day. Type is divided into non-dynamic and dynamic, 
non-dynamic vacuum disc is not only simple structure, and low noise, but its stability is poor. A dynamic structure is more complex, but it has a high reliability, the use of more and more common. In this paper, the system needs high stability, so the selection of a dynamic vacuum sucker.

Power source selection: Vacuum sucker gas path is the use of negative pressure suction cup to inhale and exhale, the design, we must first consider the selection of power plant. At present, the power source commonly used in industry is vacuum pump and vacuum generator. Vacuum pump works: the use of mechanical, physical, chemical and other knowledge, the closure of the gas inside the vacuum and get away. With vacuum fast, high vacuum, high cost and so on. Vacuum generator works: Rapid injection of compressed air to the nozzle, the air at the mouth of the mouth quickly gathered to form compressed air, and thus the impact of volume suction flow. In the role of suction, the mouth around the mouth of the air continue to suck out, and thus have a certain degree of vacuum. As the set of automatic film forming machines require compact structure, high efficiency, so the choice of vacuum generator for the vacuum system power source.

The basic formula of the vacuum generator: From the principle of the vacuum generator, in the fluid mechanics, the continuity equation of the incompressible air is:

$$
A_{1} \cdot v_{1}=A_{2} \cdot v_{2}
$$

Where, $A_{1}, A_{2}$ for the pipe cross-sectional area, $v_{1}, v_{2}$ for the air flow rate.

According to Bernoulli's ideal equation:

$$
p_{1}+1 / 2 p v_{1}^{2}=p_{2}+1 / 2 p v_{2}^{2}
$$

Where, p1,p2for the cross-section of the corresponding A1,A2, V1,V2 pressure for the corresponding cross-section A1,A2 of the flow rate, $\rho$ is the air density.

Air compressor selection: air compressor according to the work of different, divided into piston type, vane type and screw type.

Air compressor according to the work of different, divided into piston type, vane type and screw type. Which piston and vane air compressor working environment is more demanding, more suitable for use in the laboratory environment. In this system, through the comparative analysis, select the high cost of screw air compressor.

Output pressure is calculated as:

$$
p_{c}=p+\sum \Delta p
$$

Where $\mathrm{p}$ is the maximum use of pneumatic components, $\sum \Delta \mathrm{p}$ general election 2.015.0 Mpa.

Air compressor inlet air volume formula:

$$
q_{c}=k q_{b} q_{s a}
$$

Where $\mathrm{qb}_{\mathrm{b}}$ is the need to provide the amount of gas to the pneumatic system, $\mathrm{q}_{\mathrm{sa}}$ is the average air consumption for the pneumatic system, $\mathrm{k}$ is the correction factor, generally take 2 to 5.1.

This chapter mainly analyzes the pneumatic system, focusing on the cylinder, vacuum suction cups and air compressor selection method. And the principle of aerodynamics was explained, for the further analysis of the accumulation of experience in pneumatic systems.

\section{Servo Motor Selection}

Servo motor selection than ordinary motor selection is much more complex, generally need to consider the following points.

(1)Load motor inertia ratio: Normally, the drive has a parameter to indicate the ratio of the moment of inertia of the load to the moment of inertia of the motor. The appropriate inertia in the system can fully tap the servo control system performance. Servo motor to achieve the best working condition, inertia ratio of the critical value of 20 . Servo system performance, with the inertia ratio increases, will become more and more unstable. If an unmatched inertia ratio is used, the device 
will overshoot during operation.

To calculate the inertia of the rotating workpiece:

$$
J=1 / 8 W D^{2}
$$

Where, $\mathrm{W}$ is the mass, in $\mathrm{kg}$; $\mathrm{D}$ outer diameter, the unit is $\mathrm{m}$

For stepped workpieces, it can be decomposed into the sum of each part of the total work piece made up of a number of steps:

$$
J=\frac{1}{8}\left(W_{1} D_{1}^{2}+W_{2} D_{2}^{2}+\ldots \ldots+W_{i} D_{i}^{2}\right)
$$

Inertia calculated when the hollow cylinder revolves around the central axis:

$$
J=\frac{1}{8}\left(D^{2}+d^{2}\right)
$$

There are many such cases, such as for roller, in order to reduce inertia and weight, remove some material.

Where, $\mathrm{J}$ is the inertia, in $\mathrm{kg}$; $\mathrm{W}$ mass, in $\mathrm{kg}$; $\mathrm{D}$ is the outer diameter, in $\mathrm{m}$; $\mathrm{d}$ is the inner diameter, the unit is $\mathrm{m}$.

Calculation of Inertia Induction:

$$
J_{2}=j_{1}\left(\frac{n_{2}}{n_{1}}\right)^{2}
$$

Where $\mathrm{j}_{1}$ is the inertia of 1 , in $\mathrm{kg} \cdot \mathrm{m} 2 ; \mathrm{J}_{2}$ is the inertia of 2 , in $\mathrm{kg} \cdot \mathrm{m} ; \mathrm{n}_{1}$ is the speed of 1 , in units of $\mathrm{r} / \mathrm{min} ; \mathrm{n}_{2}$ is 2 speed, unit of $\mathrm{r} / \mathrm{min}$. The function of the formula is to convert the inertia of 1 to 2 above.

Horizontal inertia calculation:

$$
J=\frac{1}{4} W D^{2}
$$

Where, $\mathrm{W}$ is the mass, in $\mathrm{kg}, \mathrm{D}$ is the diameter, the unit is $\mathrm{m}$. The servomechanism must be analyzed qualitatively according to the part design requirements. Workpiece mass WA $=5.01$ $[\mathrm{kg}]$ ( Diameter $\quad \mathrm{P}_{\mathrm{D}}=0.06[\mathrm{~m}]$; Quality $\quad \mathrm{W}_{\mathrm{P}}=0.55[\mathrm{~kg}]$; Inertia $\quad \mathrm{J}_{\mathrm{C}}=0 \quad$ )

$$
J_{1}=J_{C}+\frac{1}{4} W_{A} \times P_{D}{ }^{2}+\frac{1}{8} W_{p} \times P_{D}{ }^{2} \times 2
$$

$\mathrm{J}_{1}=68.8 \times 10^{-4}$, the unit is $10^{-4} \mathrm{Kg} \cdot \mathrm{m}^{2}$

As a result of the use of 1 to 6.5 reducer design, using the formula (4-4), the servo motor $\mathrm{J}_{1}$ about 11.6 units, the unit is $10^{-4} \mathrm{Kg} . \mathrm{m}^{2}$

The following is the analysis of inertia matching problem:

$$
T_{M}-T_{L}=\left(J_{M}+J_{L}\right) \alpha
$$

( $T_{M} \longrightarrow$ Output torque; $\quad T_{L} \mathrm{Load}_{-}$torque: $J_{M} \longrightarrow$ Moment of inertia $J_{L} \longrightarrow$ Load Moment of Inertia $\alpha \_$Angular acceleration)

When the primary servo motor MHMD042G1U2 is selected, its rotor moment of inertia $\mathrm{J}_{\text {Mis }} 0.71$. $\mathrm{J}_{\mathrm{L}} / \mathrm{J}_{\mathrm{M}}=11.5 / 0.7=16.5$ times, 16.5 less than 20 , to meet the requirements.

(2)Rotating speed

By the formula:

$$
N=\frac{V}{\pi P_{D}} \times n
$$

Where: $\mathrm{N}$ is the speed; $\mathrm{V}$ is the linear velocity in $\mathrm{m} / \mathrm{s} ; \mathrm{PD}_{\mathrm{D}}$ is diameter, unit is $\mathrm{m} ; \mathrm{n}$ is the 
reduction ratio. After the calculation of $\mathrm{N}$ about 22.1r / s, MHMD042G1U2 rated speed of $60 \mathrm{r} / \mathrm{s}$, $22 \mathrm{r} / \mathrm{s}<60 \mathrm{r} / \mathrm{s}$, required to achieve.

\section{(3)Torque}

The moving torque is:

$$
T_{f}=\frac{P_{D}}{2 \eta n}\left(g W_{A}+F\right)
$$

The diameter $P_{D}$ is $0.05 \mathrm{~m}, \eta$ is the efficiency, the value is $0.81 ; \mathrm{n}$ is the reduction ratio, its value is 6; $\mathrm{W}_{\mathrm{A}}$ is the load quality. Calculated, 0.255 , in units of $\mathrm{N} \cdot \mathrm{m}$.

The acceleration torque is:

$$
T_{a}=\frac{\left(J_{L}+J_{M}\right) \times 2 \pi N}{T_{1}}+T_{f}
$$

$\mathrm{J}_{\mathrm{M}}$ is the load inertia, $\mathrm{J}_{\mathrm{M}}$ value is 11.6 , the unit is $10^{-4} \mathrm{Kg}^{2} \mathrm{~m}^{2} ; \mathrm{N}$ is the rotational speed, its value is $22.01 \mathrm{r} / \mathrm{s}$; The $\mathrm{T}_{1}$ is the inertia, $\mathrm{J}_{\mathrm{M}}$ is the inertia, the $\mathrm{J}_{\mathrm{L}}$ value is 0.71 , the unit is $10^{-4} \mathrm{Kg}^{2} \mathrm{~m}^{2}$; For the acceleration time, which is $0.2 \mathrm{~s}$. Calculate the system to accelerate the movement required torque Ta 1.1, in units of $\mathrm{N} \cdot \mathrm{m}$.

The deceleration torque is:

$$
T_{d}=\frac{\left(J_{L}+J_{M}\right) \times 2 \pi N}{T_{2}}-T_{f}
$$

Where $T_{L}$ the deceleration torque for the servo system is 0.7 and the unit is $N \cdot m$. By $T_{f}, T_{a}, T_{b}$ The servo system can calculate the calculated torque of $1.1 \mathrm{~N} \cdot \mathrm{m}$.

\section{Conclusion}

This paper describes the mechanical structure of the generation of automatic molding machine, describes the SolidWorks three-dimensional modeling and the automatic bagging machine structure of the design and function of the description, analysis of the pneumatic system, the pneumatic principle was carried out. The calculation and the experience for the analysis of the pneumatic system have been accumulated, and the design and calculation of the servo system preliminary selection have been carried out.

\section{References}

1. Onaqa E.High speed packaging robot [J].Robot, 1997(119):12-17.

2. Kim Y H, Huff-Lonergan E, Sebranek J G, et al. High-oxygen modified atmosphere packaging system induces lipid and myoglobin oxidation and protein polymerization [J]. Meat science, 2010, 85(4): 759-767.

3. Bauer N, Engell S, Huuck R, et al. Verification of PLC programs given as sequential function charts[M].Integration of Software Specification Techniques for Applications in Engineering. Springer Berlin Heidelberg, 2004: 517-540.0

4. Ito Y, Yoshida Y. Design Conception of Hierarchical Modular Construction-manufacturing Different Kinds of Machine Tools by Using Common Modules[C]. Proc. 19th Int. MTDR Conf., Mac-Millan. 1979,147.

5. Chen Yin, activity based on virtual PLC castor automatic assembly machine modeling and simulation [D]. Master thesis of guangdong university of technology, 2012

6. Hillstrom F. Applying axiomatic design to interface analysis in modular product development [J]. Advances in Design Automation, 1994, 69(2): 363-37 
7. Ito Y, Yoshida Y. Design Conception of Hierarchical Modular Construction-manufacturing Different Kinds of Machine Tools by Using Common Modules[C]. Proc. 19th Int. MTDR Conf., Mac-Millan. 1979,147.

8. Ziemba R. Use of a programmable logic controller (PLC) for temperature, position, velocity and pressure control of injection molding machinery[C]. Industry Applications Society Annual Meeting, 1988. Conference Record of the 1988.IEEE, 1988:1397-1404.

9. Xueming Z, Guohou L, Feng S, et al. Application of fuzzy control in the servo system[C]. Proceedings of the 21st annual international conference on Chinese Control and Decision Conference. IEEE Press, 2009:1696-1699

10. Cai T F, Zhou J Z, Wu X M, et al. Monitor System for Automatic Grouting of Metamorphic Concrete Based on PLC and HMI [J]. Applied Mechanics and Materials, 2013, 365: 662-665. 